\title{
Aspects réglementaires de la petite hydroélectricité
}

\author{
Law aspects of small hydropower
}

\author{
par A. Delaunay \\ Direction de l'Eau du ministère de l'Aménagement du territoire et de l'Environnement
}

The 1992 water act (french law of January 3, 1992) appreciably modifies the prescribed framework proceeding from the 1919 law on use of hydroelectric power. A single procedure has been made for one application of both 1919 \& 1992 laws. The master water development and management plans ( "SDAGE " : schémas directeurs d'aménagement et de gestion des eaux) fixed the basic trends of balanced management of the water resource at basin catchment area level. The aim of the ministry is to conciliate how to modernize a renewable energy and the balanced management of the water resource, avoiding, as possible, building new dams.

\section{I $\square$ PRINCIPES GÉNÉRAUX DE LA LOI DU 16 OCTOBRE 1919}

\subsection{Historique}

L'énergie hydraulique, liée à la pente des cours d'eau, est utilisée depuis de nombreux siècles, depuis la construction des moulins à eau avec roues en bois jusqu'aux centrales hydroélectriques modernes. La police spéciale de l'énergie hydraulique a été instituée par la loi du 16 octobre 1919, relative à l'utilisation de l'énergie hydraulique.

A partir de 1850, l'utilisation de l'énergie hydraulique a été révolutionnée par plusieurs inventions : turbines hydrauliques, conduites forcées, lignes électriques assurant le transport de l'énergie et son utilisation à distance. Certaines vallées de montagne ont alors connu un essor industriel grâce à l'hydroélectricité, dénommée " houille blanche ".

Son développement rencontrait néanmoins des obstacles juridiques considérables, en raison du nombre de propriétaires concernés. En effet, le droit d'usage de l'eau et de la "pente " du cours d'eau appartenait alors à tous les riverains et l'industriel, soucieux d'équiper une chute, devait recueillir l'accord de l'ensemble des propriétaires riverains du cours d'eau entre la prise et la restitution des eaux. A défaut, il risquait de voir s'engager un recours judiciaire de ces derniers en vertu de l'article 645 du Code civil. Un certain nombre de spéculateurs, "barreurs" ou "pisteurs", n'hésitaient pas à acheter une partie ou la totalité des terrains riverains d'un cours d'eau, afin de revendre ensuite " au prix fort " les droits de riveraineté, indispensables à l'équipement de la chute.

Afin d'illustrer certains termes employés, le schéma-type d'une usine hydroélectrique avec dérivation est décrit ci-après (cas où l'usine n'est pas au pied du barrage mais à distance).

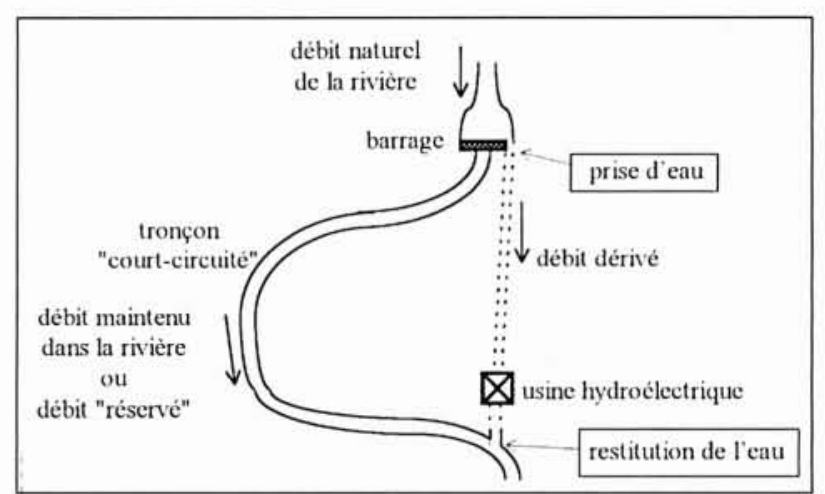

1. Schéma-type d'usine hydroélectrique avec dérivation.

\subsection{Principes de la loi du 16 octobre 1919}

Afin d'utiliser au mieux cette richesse nationale que représente l'énergie hydraulique, la loi du 16 octobre 1919 s'est inspirée de la législation relative à l'exploitation des ressources minières et dispose en son article premier que " nul ne peut disposer de l'énergie des marées, des lacs et des cours d'eau, quel que soit leur classement, sans une concession ou une autorisation de l'Etat ". Elle ne fait pas de différence entre les cours d'eau domaniaux et non domaniaux ; elle considère l'énergie hydraulique comme une richesse nationale et donne des pouvoirs étendus à l'Etat pour son exploitation.

La loi du 16 octobre 1919 précitée a institué un régime de concession pour les usines importantes et un régime d'autorisation pour les petites entreprises. La petite hydroélectricité est donc concernée essentiellement par le régime de l'autorisation (actuellement usines de moins de $4500 \mathrm{~kW}$ ), aussi seul le paragraphe suivant rappellera pour mémoire quelques caractéristiques des concessions et le reste sera exclusivement consacré aux autorisations. 


\subsection{Régime de la concession}

C'est un régime de concession de travaux publics, souvent doublé d'un régime de concession de service public lorsque l'énergie produite fait l'objet d'une distribution publique aux usagers. Le concessionnaire acquiert les terrains nécessaires au nom de l'Etat, réalise les travaux de construction du barrage et de l'usine entièrement au nom de l'Etat, dispose de certaines prérogatives de la puissance publique (possibilité d'exproprier, d'imposer des servitudes de canalisation, etc.), mais se voit imposer quelques contreparties (retour des biens à l'Etat en fin de concession, mise à disposition de réserves en eau et en force. c'est-à-dire d'une obligation de fourniture d'eau ou d'énergie électrique à prix réduit pour les services publics ou d'utilité générale, redevance financière versée à l'Etat, compensation du préjudice piscicole causé par la présence de l'ouvrage).

Font l'objet d'une concession d'une durée maximale de soixante-quinze ans les usines dont la puissance maximale brute dépasse un certain seuil : fixé par la loi du 16 octobre 1919 à 150 kilowatts pour les entreprises ayant pour objet principal la fourniture de l'énergie à des services publics, et de 500 kilowatts pour les autres, ce seuil a été uniformisé à 500 kilowatts par le décret $\mathrm{n}^{\circ} 59-60$ du 3 janvier 1959, puis porté à 4500 kilowatts par l'article 25 de la loi $n^{\circ} 80-531$ du 15 juillet 1980.

Depuis l'intervention de la loi $n^{\circ} 92-3$ du 3 janvier 1992 sur l'eau, la forme et la procédure d'instruction des demandes de concession et de déclaration d'utilité publique des ouvrages utilisant l'énergie hydraulique sont accordées à la fois au titre de la loi d'octobre 1919 et de la loi sur l'eau selon la procédure fixée par le décret $n^{\circ} 94-894$ du 13 octobre 1994. Ce décret est en cours de modification pour, d'une part, fixer les modalités de mise en concurrence pour les usines n'entrant pas dans le monopole d'EDF, d'autre part. déconcentrer au niveau du préfet l'octroi des concessions de moins de $100 \mathrm{MW}$.

Parallèlement, le cahier des charges-type fixé par le décret du 5 septembre 1920 est en cours de révision.

Le changement de seuil de concession en 1980, de 500 à $4500 \mathrm{~kW}$ pose un problème spécifique lors de l'échéance des titres des concessions concernées. En effet, les biens font retour dans le domaine hydroélectrique de l'Etat à l'échéance de la concession et, s'agissant d'usines de moins de $4500 \mathrm{~kW}$, ces usines ne seront pas à nouveau concédées. Sur les cours d'eau non domaniaux, le principe a été arrêté que l'Etat vendrait ces usines, avec bien entendu une priorité à l'ancien exploitant. Une autorisation d'utiliser l'énergie sera instruite. Sur les cours d'eau domaniaux, les problèmes posés par la superposition du domaine concédé et du domaine public fluvial sont encore à l'étude.

\subsection{Régime de l'autorisation}

Le régime d'autorisation institué par la loi du 16 octobre 1919 dépasse un régime de simple police et concerne l'utilisation de l'énergie hydraulique, considérée comme une richesse nationale.

Ainsi le préfet du Puy-de-Dôme a pu valablement autoriser une microcentrale malgré l'opposition supposée de nombreux riverains car, d'une part, "l'établissement d'une usine électrique d'environ $400 \mathrm{~kW}$ de puissance utile, dont la production est destinée à être distribuée aux usagers par le réseau d'EDF, présente à cet égard un caractère d'utilité générale " et. d'autre part. " les prescriptions techniques imposées au bénéficiaire de l'autorisation sont suffisantes pour assurer la sauvegarde des paysages et la protection de la pisciculture" (Cons. Etat., 30 avril 1982. Assoc. " Sauvons nos rivières ").

Par contre, l'Etat a pour mission de faire respecter les intérêts généraux énumérés à l'article 10 de la loi du 16 octobre 1919 précitée et peut refuser une demande d'autorisation sur cette base, sous réserve que l'arrêté de refus soit précisément motivé.

Ainsi, le préfet de Haute-Loire était fondé à refuser une autorisation d'usine hydraulique en se fondant sur des considérations tirées de la protection de l'environnement : l'installation n'aurait laissé subsister sur une partie centrale du cours d'eau d'environ mille mètres, constituée d'une série de biefs rocheux, qu'un débit insuffisant pour assurer de façon permanente la circulation des poissons et le pétitionnaire n'a proposé aucune mesure compensatoire (Cons. Etat., 10 février 1995, n 108340, Sec. Et. Environnement c/ M. Georges Alizert).

L'Etat a également en charge d'assurer le meilleur aménagement énergétique des cours d'eau. Une autorisation peut être refusée, ou limitée dans sa durée, si elle peut contrarier ce bon aménagement énergétique, par exemple en n'utilisant que partiellement les potentialités d'un site ou en risquant de gêner l'octroi d'une future concession de puissance très supérieure. De même, lorsque plusieurs demandes concurrentes sont présentées sur le même site, ou sur des sites proches, l'administration doit choisir la demande qu'elle estime devoir assurer la meilleure utilisation énergétique du cours d'eau, et rejeter les autres demandes.

\subsection{Dispositions spéciales liées au changement de seuil de concessibilité opéré par la loi de $\mathbf{1 9 8 0}$}

La loi ${ }^{\circ} 80-531$ du 15 juillet 1980 a porté le seuil de concessibilité de 500 à 4500 kilowatts, dans le souci de simplifier la réglementation applicable à ces usines et ainsi favoriser leur développement. Toutefois, cette loi a prévu le maintien des obligations en matière de fourniture d'énergie réservée pour ces usines de puissance comprise entre 500 et 4500 kilowatts.

Elle a également instauré la possibilité d'exproprier les terrains ou de les grever de servitudes pour les collectivités locales ou leur groupement, lorsqu'elles aménagent et exploitent directement une usine hydraulique soumise à autorisation.

\section{- 1.6. Rivières " réservées"}

La loi du 15 juillet 1980 a également introduit la notion de rivières dites " réservées ". L'article 2 de la loi du 16 octobre, ainsi modifié, prévoit ainsi que " sur certains cours d'eau dont la liste sera fixée par décret en Conseil d'Etat ", d'une part, aucune autorisation ou concession ne sera donnée pour des entreprises hydrauliques nouvelles et, d'autre part. pour les entreprises existantes qui satisfont aux dispositions des articles 25 ou 27 de la loi $n^{\circ} 80-531$ du 15 juillet 1981 , * une concession pourra être accordée sous réserve que la hauteur du barrage ne soit pas modifiée ".

En effet, les parties de cours d'eau restées dans un état proche d'un état naturel, ou ne subissant pas les effets d'aménagements (tronçons court-circuités ou soumis à des éclusées), sont devenus très rares et représentent moins de $10 \%$ du linéaire des cours d'eau français. La préservation de ces cours d'eau est essentielle du point de vue patrimonial 
ainsi que pour permettre d'étudier l'impact réel des aménagements par comparaison entre le cours d'eau aménagé et un cours d'eau naturel comparable. L'expérience montre qu'il devient très difficile de trouver de tels points de références. Il est d'autant plus essentiel de les préserver.

La présence de très nombreux aménagements sur certaines parties de cours d'eau a également justifié leur classement afin de préserver le potentiel biologique résiduel. Ce classement n'empêche pas la modernisation des usines régulièrement existantes, sous réserve de ne pas augmenter la hauteur des barrages.

Les propositions de classement sont adressées au ministère de l'Aménagement du Territoire et de l'Environnement par les préfets après avis du Conseil Général. Si la procédure est avancée dans certains départements, dans d'autres, elle est encore au stade d'instruction locale.

\subsection{Apports de la loi pêche du 30 juin 1984}

La loi n84-514 du 29 juin 1984 sur la pêche a apporté des améliorations pour l'insertion environnementale des microcentrales, dont les principales sont les suivantes :

- le débit maintenu dans la rivière à l'aval du barrage doit être suffisant pour garantir en permanence la vie, la circulation et la reproduction des espèces piscicoles conformément à l'article L. 232-5 du Code rural. Il ne peut être inférieur à $10 \%$ du débit moyen annuel du cours d'eau, ou module, pour les ouvrages nouveaux ou lors du renouvellement des concessions ou autorisations. Les ouvrages régulièrement existant au 30 juin 1984 doivent se rapprocher progressivement de cette valeur avec une première échéance fixée au 30 juin 1987 pour atteindre le quarantième du module.

- sur certains cours classés au titre de l'article L. 232-6 du code rural, tout ouvrage doit comporter des dispositifs assurant la circulation des poissons migrateurs. L'exploitant de l'ouvrage est tenu d'assurer le fonctionnement et l'entretien de ces dispositifs.

- le préfet peut exiger qu'Electricité de France n'achète plus l'électricité produite par une microcentrale irrégulièrement installée ou ne respectant pas les prescriptions imposées.

\section{II — LES APPORTS DE LA LOI SUR L'EAU À LA POLICE DE L'ÉNERGIE HYDRAULIQUE}

\subsection{Principes d'application de la loi sur l'eau aux usines hydroélectriques}

L'article 10-V de la loi $\mathrm{n}^{\circ} 92-3$ du 3 janvier 1992 sur l'eau précise que " les règlements d'eau des entreprises hydroélectriques sont pris conjointement au titre de l'article 10 de la loi du 16 octobre 1919 relative à l'utilisation de l'énergie hydraulique et du présent article. Ces règlements peuvent faire l'objet de modifications, sans toutefois remettre en cause l'équilibre général de la concession."

Les décrets d'application communs aux deux lois sont ainsi, d'une part, le décret n $94-894$ du 13 octobre 1994 précité pour les concessions, d'autre part, le décret n ${ }^{\circ}$ 95-1204 du 6 novembre 1995 pour les autorisations. Ce dernier sera commenté ci-dessous.

\subsection{SDAGE et SAGE}

Les Schémas Directeurs d'Aménagement et de Gestion des Eaux (SDAGE) ont été élaborés par les comités de bassins et approuvés dans chacun des six grands bassins hydro- graphiques français. Ils ont défini des orientations fondamentales pour la gestion équilibrée de l'eau et la protection des milieux aquatiques. Ils ont notamment signalé des rivières ou des milieux aquatiques remarquables à protéger plus particulièrement, des axes importants pour la circulation des poissons migrateurs, préconisé le maintien de débits biologiques dans les cours d'eau, etc.

Toutes les autorisations dans le domaine de l'eau, dont celles d'utiliser l'énergie hydraulique, doivent être compatibles, ou rendues compatibles, avec le SDAGE.

Ces SDAGE peuvent être prolongés au niveau d'un sousbassin hydrographique par un Schéma d'Aménagement et de Gestion des Eaux (SAGE). Celui-ci est élaboré par une commission locale de l'eau comprenant des élus, des usagers de l'eau et l'administration. Il permet de dégager des orientations locales pour une gestion équilibrée de la ressource en eau.

\subsection{Renouvellements des autorisations et arrêtés com- plémentaires}

L'article 47 de la loi du 3 janvier 1992 sur l'eau, directement applicable, a modifié les articles 13,16 et 18 de la loi du 16 octobre 1919. Il a modifié les délais de préavis en cas de demande de renouvellement (onze ans pour les concessions et cinq ans pour les autorisations), ainsi que la date à laquelle l'administration doit informer le pétitionnaire de sa décision d'accorder ou non un nouveau titre (cinq ans pour les concessions et trois ans pour les autorisations). Faute de décision de l'administration, la durée de validité du titre est prolongée aux conditions antérieures, pour une durée équivalente au retard de l'administration.

La possibilité de renouvellement tacite pour trente ans, qui était auparavant prévue en cas d'absence de décision de l'administration en temps voulu, est supprimée. Tout renouvellement doit donc expliciter les nouvelles conditions de fonctionnement des ouvrages en prenant en considération à la fois l'intérêt économique de l'exploitation, d'une part, et l'équilibre des milieux aquatiques, d'autre part. Cela permet un réexamen des conditions d'exploitation d'ouvrages, afin de mieux les insérer dans leur environnement. En effet, un renouvellement est juridiquement considéré comme une nouvelle autorisation et, les ouvrages ayant été amortis sur la période précédente, le pétitionnaire peut s'engager en toute connaissance de cause en fonction des conditions de la nouvelle autorisation. Le renouvellement est ainsi l'occasion d'une remise à niveau des installations (débit réservé, dispositifs de franchissement des poissons migrateurs, paysage, etc.).

La mise en cuvre des arrêtés complémentaires est également une possibilité ouverte au préfet pour adapter les conditions d'exploitation d'usines dont le titre est en cours de validité afin d'assurer la gestion équilibrée de la ressource en eau, notamment en cas d'impact majeur sur le milieu aquatique visé à l'article 10-IV de la loi sur l'eau. Ces arrêtés complémentaires sont pris par le préfet après avis du conseil départemental d'hygiène, l'exploitant pouvant y être entendu. Ils peuvent également être pris pour rendre l'autorisation compatible avec le SDAGE ou un SAGE, à condition de ne pas remettre en cause l'équilibre général de l'exploitation. Il est en effet logique que cette adaptation d'un ouvrage non financièrement amorti, ne doive pas se traduire par un bouleversement de l'équilibre entre dépenses et recettes, au risque de rendre l'entreprise déficitaire.

Enfin, l'article 18 de la loi du 16 octobre 1919 précitée avait prévu que les usines autorisées avant son entrée en 
vigueur deviendraient automatiquement la propriété de l'Etat à la fin du régime provisoire de soixante-quinze ans. L'article 47 de la loi du 3 janvier 1992 précitée sur l'eau a modifié cet article et a logiquement limité ce retour à l'Etat aux seules entreprises concessibles, c'est-à-dire actuellement celles d'une puissance supérieure à 4500 kilowatts.

\subsection{Politique du ministère de l'Environnement en matière de microcentrales}

L'utilisation de l'énergie hydraulique assure une part non négligeable de la production d'électricité par rapport à la filière nucléaire ou thermique classique, notamment en ce qui concerne la production de pointe. Chaque type de production génère des effets, très variables d'une filière à l'autre, sur l'environnement, au regard de l'effet de serre, de la santé, de l'impact sur l'eau et le milieu aquatique, de la gestion des sous-produits et déchets ou de la remise en état des sites à la fin de la période de production. L'hydroélectricité peut présenter des atouts vis-à-vis du thermique au regard de l'effet de serre sous réserve d'une maitrise des effets sur le milieu aquatique. Pour éviter ces distorsions de traitement entre les différentes filières énergétiques, il est nécessaire d'internaliser les différents coûts externes supportés par la collectivité.

L'utilisation de l'énergie hydraulique a permis un développement économique important dans certaines vallées de montagne. L'énergie hydraulique a été très développée en France et la plupart des cours d'eau ont été largement équipés depuis un siècle, alors que les préoccupations d'environnement, plus récentes, concernent essentiellement les nouvelles constructions. Peu de cours d'eau sont restés dans un état proche d'un état naturel ce qui nous prive de références pour apprécier l'impact réel des aménagements. De nombreuses autres activités humaines (déversement d'eaux usées, usages industriels ou agricoles, navigation, tourisme, extractions de granulats, etc.) ont également contribué à une dégradation des cours d'eau.

C'est dans ce contexte actuel d'importance des secteurs dégradés, que le ministère de l'Aménagement du Territoire et de l'Environnement s'est donné comme priorité l'amélioration des conditions de fonctionnement du parc existant. Son objectif passe par la conciliation de la modernisation d'une énergie renouvelable et de la gestion équilibrée de la ressource en eau, en évitant autant que possible la construction de nouveaux barrages. Le renouvellement des titres anciens, la mise en cuvre d'arrêtés complémentaires, l'application des SDAGE et des SAGE sont les moyens pour l'atteindre.

\subsection{Procédure d'autorisation}

\subsubsection{Principe du décret $n^{\circ} 95-1204$ du 6 novembre 1995}

La procédure d'instruction des demandes d'autorisation d'ouvrages utilisant l'énergie hydraulique est fixée par le décret $n^{\circ}$ 95-1204 du 6 novembre 1995. C'est la même procédure que pour tous les autres ouvrages hydrauliques soumis à autorisation avec seulement quelques adaptations aux spécificités de l'hydroélectricité, notamment en matière d'énergie réservée. La procédure n'est donc pas plus lourde pour l'hydroélectricité que pour les autres ouvrages hydrauliques. Le faible nombre d'autorisations délivrées dépend plus de facteurs économiques, et du fait que les sites les meilleurs ont déjà été équipés, que de la longueur de la procédure.

Ainsi, les demandes seront instruites selon la procédure générale instituée par le décret n 93-742 du 29 mars 1993 , sous réserve de quelques compléments ou dérogations justifiés par la spécificité de la police de l'énergie hydraulique, à laquelle s'associe la gestion d'une ressource nationale.

\subsubsection{Adaptation du dossier de demande d'autorisation}

Un dossier spécifique de demande d'autorisation est demandé, comprenant notamment divers plans, des justifications des capacités techniques et financières du pétitionnaire, une étude d'impact lorsque la puissance dépasse 500 kilowatts, ou une notice d'impact dans les autres cas, les accords éventuels concernant la fourniture d'énergie réservée, ainsi que le cas échéant pour les collectivités locales sollicitant la déclaration d'utilité publique des travaux, divers plans, notes ou avis concernant les servitudes ou expropriations envisagées.

\subsubsection{Consultations}

L’avis du conseil général est demandé, notamment en ce qui concerne l'énergie réservée. L'avis du conseil départemental d'hygiène est requis dans tous les cas, et celui de la commission des sites, perspectives et paysages uniquement lorsque les ouvrages concernent un site classé ou inscrit au titre de la loi du 2 mai 1930. Cela limite les cas de double saisine de commissions administratives au niveau départemental, tout en permettant au conseil départemental d'hygiène d'avoir une vision globale des aménagements de cours d'eau.

\subsubsection{Demandes concurrentes}

En outre, le décret fixe la procédure et les délais pour choisir, parmi d'éventuelles demandes concurrentes, celle que l'administration estime devoir assurer le meilleur aménagement énergétique du cours d'eau. Le délai global donné au préfet pour instruire le dossier après la clôture de l'enquête publique est porté de trois à six mois, avec prorogation éventuelle de deux mois, soit un maximum de huit mois.

Il est à noter que bien qu'il s'agisse de l'exploitation d'une richesse nationale, les autorisations hydroélectriques ne sont pas considérées comme des délégations de service public et la loi Sapin ne leur est pas applicable.

\subsubsection{Plans des caractéristiques générales}

Par ailleurs, le décret $\mathrm{n}^{\circ}$ 95-1204 du 6 novembre 1995 introduit un stade d'approbation des plans fixant les caractéristiques générales des ouvrages. En effet, lorsqu'il y a barrage de cours d'eau, l'autorisation est accordée sur la base d'un avant-projet sommaire, et le pétitionnaire n'engage les dépenses d'études détaillées, notamment pour les fondations sur le sous-sol, qu'une fois en possession de son autorisation. Des modifications de détail interviennent souvent sans remettre en cause la consistance de l'usine (donc ne touchant pas à la cote d'exploitation du plan d'eau, à la cote de restitution des eaux, ou au débit maximal dérivé). Cela justifie un examen par l'administration de la conformité des projets définitifs avec l'autorisation. Toutefois, pour des ouvrages simples et si ces plans détaillés sont fournis dans le dossier initial, rien ne s'oppose à ce que le même arrêté préfectoral délivre l'autorisation et approuve ces plans détaillés.

\subsubsection{Récolement des travaux}

Le décret $n^{\circ}$ 95-1204 du 6 novembre 1995 prévoit également une procédure de récolement des travaux, laquelle permet de vérifier, après leur achèvement, si ceux-ci sont conformes à l'autorisation. Cette procédure existait déjà dans le décret $n^{\circ} 81-375$ du 15 avril 1981, mais n’a pas été instituée de façon systématique dans la procédure générale fixée

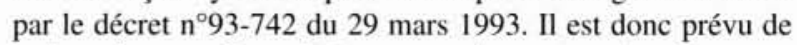
maintenir cette formalité en ce qui concerne les ouvrages hydroélectriques. 


\subsubsection{Durée des autorisations}

La durée des autorisations ou concessions hydroélectriques ne peut excéder 75 ans, délai maximal fixé par la loi du 16 octobre 1919. Toutefois, le délai d'amortissement financier des ouvrages est généralement moindre et la durée préconisée actuellement par le ministère de l'Aménagement du Territoire et de l'Environnement est de trente ans pour les autorisations d'ouvrages hydroélectriques (quarante ans en moyenne pour les concessions). Une stabilité juridique est en effet nécessaire compte tenu de l'importance des investissements réalisés.

Par contre, ces autorisations doivent pouvoir être adaptées pendant la durée de l'autorisation en utilisant les meilleurs techniques environnementales à un coût économiquement acceptable. Notamment, le règlement d'eau de l'usine doit être modifié par arrêté complémentaire lorsque c'est nécessaire pour sauvegarder les intérêts généraux mentionnés à l'article 2 de la loi du 3 janvier 1992 sur l'eau tels que la sécurité publique ou la préservation des écosystèmes aquatiques. Toutefois, ces modifications ne doivent pas bouleverser l'équilibre financier de l'autorisation, au point d'en remettre en cause l'équilibre général.

Ainsi, le ministère de l'Aménagement du Territoire et de l'Environnement demande qu'un suivi de l'impact réel de l'ouvrage sur le milieu aquatique soit mené. L'importance de ce suivi doit être adaptée à la taille de l'ouvrage et à la sensibilité du milieu. Il peut être allégé si l'ouvrage existe depuis longtemps et si son incidence est déjà bien connue, ou, au contraire, renforcé sur un ouvrage nouveau dont le document d'incidences laisse apparaître des incertitudes sur son impact réel. A l'issue du suivi, le préfet peut modifier par arrêté complémentaire les prescriptions de l'arrêté, en fonction de l'impact réel constaté par rapport à l'impact initialement prévu.

Le préfet dispose également de la faculté de soumettre, dès l'autorisation initiale, certaines dispositions à réexamen périodique en vertu de l'article 17 du décret $\mathrm{n}^{\circ}$ 93-742 du 29 mars 1993 susvisé. Cette solution de réexamen de certaines dispositions techniques assorti d'une durée d'autorisation de 30 ans paraît préférable à l'octroi d'une autorisation de trop courte durée. Pour l'exploitant, la mise à jour du document d'incidence est limitée aux aspects essentiels et les formalités sont allégées (pas d'enquête publique). Pour le préfet, les prescriptions techniques sont réexaminées, et en cas de refus de l'exploitant de procéder au réexamen, après mise en demeure restée infructueuse, il peut engager une procédure de déchéance.

\subsubsection{Entrée en vigueur du décret}

Le décret se substitue au décret $n^{\circ}$ 81-375 du 15 avril 1981 et l'abroge. Toutefois, en application de l'article 78 de la loi $n^{\circ} 95-101$ du 2 février 1995 relative au renforcement de la protection de l'environnement, les procédures engagées sur la base du décret du 15 avril 1981 précité pourront être poursuivies jusqu'à leur achèvement selon la procédure fixée par ledit décret, et l'autorisation délivrée à ce titre vaut également autorisation au titre de l'article 10 de la loi ${ }^{\circ} 92-3$ du 3 janvier 1992 sur l'eau. L'engagement de la procédure est constaté par l'accusé de réception du préfet d'un dossier régulier et complet, prévu par l'article 5 du décret du 15 avril 1981 précité.

\subsubsection{Application aux autorisations antérieurement délivrées}

Les autorisations antérieurement délivrées, c'est-à-dire celles délivrées en application du décret $\mathrm{n}^{\circ}$ 81-375 du 15 avril 1981, ou des textes auxquels il s'est substitué, ou encore celles de moins de 150 kilowatts délivrées avant le
16 octobre 1919, sont assimilées, pour les installations existantes, aux autorisations délivrées au titre du décret $\mathrm{n}^{\circ}$ 95-1204 du 6 novembre 1995. Elles ne nécessitent donc pas de nouvelle procédure d'autorisation mais elles peuvent être modifiées selon cette procédure, soit à la demande de l'exploitant, soit à l'initiative du préfet dans l'intérêt de la gestion équilibrée de la ressource en eau, pour la préservation de la salubrité publique, la prévention des inondations ou rendre l'autorisation compatible avec un SDAGE ou un SAGE.

Les ouvrages fondés en titre, pouvant prouver leur existence légale et n'ayant pas augmenté leur puissance, ne nécessitent pas non plus d'autorisation nouvelle. Ils peuvent toutefois être réglementés en application de l'article 10-IV de la loi sur l'eau ou de l'article 109 du code rural en vue de préserver la salubrité publique ou de prévenir les inondations en assurant l'écoulement normal des eaux.

Les modifications de ces ouvrages, ainsi que celles des ouvrages fondés en titre, ou les changements apportés à leur mode d'utilisation, sont instruits selon la nouvelle procédure. Ainsi, en application de l'article 15 du décret n ${ }^{\circ} 93-742$ du 29 mars 1993, au vu de la déclaration d'intention du pétitionnaire et selon l'incidence prévisible sur le milieu aquatique des modifications des ouvrages ou de leur mode d'utilisation, le préfet peut, s'il y a lieu, fixer des prescriptions complémentaires après avis du conseil départemental d'hygiène, ou exiger le dépôt d'une nouvelle demande d'autorisation.

\subsection{Règlement d'eau-type}

Le décret $n^{\circ}$ 95-1205 du 6 novembre 1995 a fixé un nouveau règlement d'eau-type et a abrogé le décret $n^{\circ}$ 81-376 du 15 avril 1981. La publication d'un règlement d'eau-type, dont le principe avait été prévu par la loi du 16 octobre 1919 , a pour avantage d'uniformiser les règles et la présentation des différentes autorisations d'un département à l'autre et d'éviter ainsi d'éventuelles distorsions de concurrence entre producteurs.

Ce règlement d'eau-type, adaptant les modèles précédents, fixe un cadre complet pour l'autorisation. Les notes de fin de texte explicitent les mentions à apporter, certaines par exemple ne concernant que les cours d'eau domaniaux, ou d'autres, à l'inverse, les cours d'eau non domaniaux. Il complète le décret relatif à la procédure (approbation des plans, récolement, etc.). Dans le domaine de la compensation des atteintes que la présence et le fonctionnement de l'ouvrage apportent à la vie, à la circulation et à la reproduction des espèces de poissons, ainsi qu'au milieu aquatique, il privilégie les actions de restauration du milieu par rapport aux alevinages.

\section{BIBLIOGRAPHIE}

[1] Ministère de l'Environnement- direction de l'eau - document d'information -Eléments d'aide à la mise en cuvre des décrets n ${ }^{\circ} 93-742$ et n ${ }^{\circ} 93$ 743 du 29 mars 1993 relatifs à l'application de l'article 10 de la loi sur l'eau - tome 1, 1994 Juin.

[2] idem - tome 2, Décembre 1994

[3] idem - tome 3, 1995 Sept.

[4] Ministère de l'Environnement- direction de l'eau - Entreprises hydroélectriques - recueil de textes, Décembre 1995

[5] Ministère de l'environnement- direction de l'eau - Entreprises hydroélectriques - Jurisprudence - Conseil d'Etat. Cours administratives d'appel, Cour de cassation, 1996 Juin

[6] Ministère de l'Aménagement du Territoire et de l'Environnement - direction de l'Eau - Document d'information - Loi sur l'eau et autres polices spéciales ayant trait à l'eau - Tome 1: Pêche et utilisation de l'énergie hydraulique, 1997 Mai 